Chirurg 2020 $91: 678$

https://doi.org/10.1007/s00104-020-01233-4

Online publiziert: 3. Juli 2020

(c) Springer Medizin Verlag GmbH, ein Teil von Springer Nature 2020

\section{Originalpublikation}

Dueland $S$, Syversveen T, Solheim JM, Solberg S, Grut H, Bjørnbeth BA, Hagness M, Line PD (2020) Survival following liver transplantation for patients with nonresectable liveronly colorectal metastases. Annals of Surgery 271(2):212-218. https://doi.org/10.1097/SLA. 0000000000003404.

Einleitung. Die Leberresektion stellt die einzige kurative Therapieoption beim hepatisch metastasierten kolorektalen Karzinom dar. Diese ist aber nur in etwa $20 \%$ der Fälle möglich. Im Rahmen der SECAI-Studie (Secondary Cancer) konnte die 5-Jahre-Überlebenserwartung durch eine Lebertransplantation auf $60 \%$ gegenüber $10 \%$ im Falle der alleinigen palliativen Chemotherapie gesteigert werden. Die aktuelle SECA-II-Studie beschäftigte sich mit den Patientenselektionskriterien, welche evtl. mit einem vergleichbaren Outcome mit den konventionellen Indikationen für Lebertransplantationen einhergehen könnten.

Methodik. In dieser prospektiven einarmigen Studie wurden zwischen 2012 und 2016 insgesamt 15 Patienten mit ausschließlich nichtresektablen Lebermetastasen eingeschlossen. Voraussetzung war ein Ansprechen auf Chemotherapie von mindestens $10 \%$ nach RECIST-Kriterien (Response Evaluation Criteria In Solid Tumors). Ein Intervall von mindestens 1 Jahr zwischen der Diagnose und der Lebertransplantation musste gehalten werden. Zur Risikostratifikation wurden der FCRS- sowie Oslo-Score verwendet.

Aladdin Ali Deeb - Michael Ardelt - Utz Settmacher

Klinik für Allgemein-, Viszeral- und Gefäßchirurgie, Jena, Deutschland

\title{
Die Rolle der Lebertransplantation bei irresektablen kolorektalen Lebermetastasen
}

Ergebnisse. Bei medianem Follow-up von 36 Monaten ergab die KaplanMeier-Kurve eine 1-, 3- und 5-JahreÜberlebenserwartung von jeweils $100 \%$, $83 \%$ und $83 \%$. Die 1, 3 und 5 Jahre rezidivfreie Überlebenserwartung betrug jeweils 53\%, $44 \%$ und 35\%. Die pulmonale Metastasierung stellte mit $75 \%$ das häufigste Rezidiv dar, welche in den meisten Fällen gut behandelbar war. Somit betrug die 1-, 2- und 4-Jahre-Überlebenserwartung jeweils $100 \%$, $73 \%$ und $73 \%$. Im Vergleich mit SECAI-Studie bestand ein signifikanter Unterschied mehrerer Selektionskriterien, insbesondere FCRS-Score (Fong Clinical Risk Score) $p=0,028$, Oslo-Score $p=0,001$, CEA $p=0,015$.

\section{Kommentar}

Durch eine strikte Patientenselektion konnte die 5-Jahre-Überlebenserwartung auf $83 \%$ in der SECA-II-Studie im Vergleich $\mathrm{zu} 60 \%$ in der SECA-I-Studie gesteigert werden. Dies ist mit dem Outcome nach Lebertransplantation bei HCC vergleichbar, welches aktuell die konventionelle maligne Hauptindikation für Lebertransplantationen darstellt. Somit bietet die Lebertransplantation die beste Überlebenserwartung bei irresektablen kolorektalen Lebermetastasen unter Berücksichtigung strikter Selektionskriterien.

\section{Korrespondenzadresse}

PD Dr. med. habil. Dr. med. univ. Michael Ardelt

Klinik für Allgemein-, Viszeral- und Gefäßchirurgie

Am Klinikum 1, 07747 Jena, Deutschland

Michael.Ardelt@med.uni-jena.de

Interessenkonflikt. A.A. Deeb, M. Ardelt und U. Settmachergeben an, dass kein Interessenkonflikt besteht. 